\title{
EDQM Health Care Terminology
}

National Cancer Institute

\section{Source}

National Cancer Institute. EDQM Health Care Terminology. NCI Thesaurus. Code C148636.

A terminology developed by the European Directorate for the Quality of Medicines and HealthCare to provide the basis for global harmonization of pharmaceutical products in marketing authorization applications, labelling, and electronic communications in pharmacovigilance and adverse reporting. For more information, please visit: https://standardterms.edqm.eu 